\title{
Do Fish Perceive Illusory Motion?
}

\author{
Simone Gori ${ }^{1,2}$, Christian Agrillo ${ }^{3}$, Marco Dadda $^{3}$ \& Angelo Bisazza ${ }^{3}$
}

SUBJECT AREAS:

MOTION DETECTION

PERCEPTION

Received

4 June 2014

Accepted

26 August 2014

Published

23 September 2014

Correspondence and requests for materials should be addressed to

S.G. (simone.gori@ unipd.it)
'Developmental and Cognitive Neuroscience lab, Department of General Psychology, University of Padua, ${ }^{2}$ Developmental Neuropsychology Unit, Scientific Institute "E. Medea, " Bosisio Parini, Lecco, ${ }^{3}$ Comparative Psychology Research Group, Department of General Psychology, University of Padua.

Motion illusion refers to a perception of motion that is absent or different in the physical stimulus. These illusions are a powerful non-invasive tool for understanding the neurobiology of vision because they tell us, indirectly, how we process motion. There is general agreement in ascribing motion illusion to higher-level processing in the visual cortex, but debate remains about the exact role of eye movements and cortical networks in triggering it. Surprisingly, there have been no studies investigating global illusory motion evoked by static patterns in animal species other than humans. Herein, we show that fish perceive one of the most studied motion illusions, the Rotating Snakes. Fish responded similarly to real and illusory motion. The demonstration that complex global illusory motion is not restricted to humans and can be found even in species that do not have a cortex paves the way to develop animal models to study the neurobiological bases of motion perception.

o animals see the world as we see it? In the last thirty years, neuroscientists have made amazing progress in understanding the functioning of our visual system. One of the most important findings is that some features of the visual system, for example, the molecular machinery that underlies visual phototransduction, are extraordinarily similar in organisms ranging from jellyfish to humans ${ }^{1}$. Neurobiologists have found that, in some cases, even the neural circuitry that processes visual information has similar organizational principles, as shown by classical investigations of the visual cortex of humans, cats and macaques ${ }^{2,3}$.

Our visual system, however, does much more than simply reproducing the outside world: it is an active process that recreates a peculiar reality for each brain. The two-dimensional image projected on our retina is decomposed into distinct streams (e.g., orientation, shapes, color, and motion features) on which the visual areas build a plausible three-dimension reconstruction of the visual environment. Our perception of a visual stimulus can, therefore, differ considerably from its physical counterpart. For this reason, even the most striking similarity between the visual systems does not guarantee that an animal perceives a visual scene as we do. In this respect, visual illusions can be an open window into how an animal perceives the world, a powerful non-invasive tool that complements neurobiological investigation ${ }^{4}$. Research on illusions has shown, for example, that the visual systems of mammals, birds and insects follows similar principles for the segregation of objects from the background $^{5,6}$.

Motion illusion is a type of visual illusion in which we perceive motion that is different (in direction, strength, etc. ${ }^{7-12}$ ) from that present in the physical stimulus; or, even more strikingly, the perception of motion is induced by a completely static pattern ${ }^{13-15}$. As regards this latter family of stimuli, neurobiological and behavioral studies showed that both fixational eye movements ${ }^{16-18}$ and cortical processing ${ }^{19-21}$ are responsible for the perception of motion, but some outstanding questions remain unsolved. In particular, there is considerable debate among investigators as to whether gaze instability is strictly necessary to the illusory motion perception or, rather, if fixational eye movements only improve the strength of the illusion. Interestingly, not all subjects report perceiving illusory motion ${ }^{22,23}$. The origin of this polymorphic response is not entirely clear, but Fraser and Wilcox ${ }^{22}$ reported a significant parent-offspring correlation in the response to a motion illusion indicating either a genetic causation or a common environment effect. A subsequent analysis based on monozygotic-dizygotic twin comparison supported the strong genetic nature of this polymorphism. Recently Gori et al. ${ }^{24}$ showed that a specific genetic variance significantly reduce the ability of perceive illusory motion.

The literature reported motion after effects (small misperceptions of motion induced by prior exposure to opposite direction motion) or illusory reversal in motion direction due to flicker in different animals (mammals, birds and insects) ${ }^{25-28}$, but these effects can also be explained in humans without complex cortical elaboration. On the contrary, the global illusory motion experienced, for example, in the Rotating Snakes illusion (RSI) ${ }^{13}$ is a very different effect. This complex and large illusory motion may share the name "illusory motion" with other small effects, but it is, indeed, different in quality, quantity and, more importantly, in the brain structures that seemed to 
be necessary to perceive it. Surprisingly, despite long-standing interest on global motion illusion by psychologists, physiologists and neuroscientists there have been no studies reporting in non-human animals an illusion able to produce global motion induced by static patterns. A partial exception is a study of Conway et al. ${ }^{20}$ reporting that direction-selective neurons in the macaque visual cortex gave directional responses when monkeys saw a static sequence of colors characterizing the RSI (Fig 1a) that induces motion perception in humans. However, this study did not show if the recorded neuronal response reaches the conscious level because the monkeys did not perform a behavioral task of motion discrimination. Due to the extreme similarity in many aspects of vision, it would not be unexpected to discover that non-human animals, which present several stages of cortical elaboration of visual motion input, may perceive illusory motion as humans. Less obvious is to predict whether lower vertebrates, such as fish, that completely lack cortical elaboration would respond to such a complex motion illusion.

Even though fish have no cortex, they need to accurately process motion stimuli to hunt, escape predators or swim in a shoal as the accurate processing of motion information could mean the difference between life and death. On the other hand, research has shown that fish can perceive first-order (luminance-defined or Fourier) motion as well as second-order (non-Fourier) motion ${ }^{29}$. The Fourier motion is a motion characterized by a change in luminance recorded on the retina. The non-Fourier motion is defined as a motion in which the moving contour is characterized by the change in some aspects (e.g., contrast, texture, or flicker) that do not result in differences in luminance. The ability to perceive non-Fourier motion is usually attributed to higher-level processing in the primate visual cortex ${ }^{29}$. Second-order motion perceived by the fish suggests that very efficient motion processing is already present in the retina ${ }^{29}$. These results, therefore, show that lower levels in the visual pathway already processed complex types of motion before the cortex appearance in mammals ${ }^{29}$. The challenge here is to discover if motion illusions like the global rotation we perceive in the static pattern of the RSI can also be processed without a cortex.

To address these issues, in the present study we investigated the response to the RSI in two species of teleost fishes. The RSI is one of the most celebrated phenomena that show global illusory motion in a static pattern. It is a powerful variation of Fraser and Wilcox's pat$\operatorname{tern}^{22}$ and is characterized by the repetition of the following luminance sequence: black-dark gray-white-light gray-black, in a circular configuration that creates a vivid impression of slow rotation. Using computer displays, individual guppies (Poecilia reticulata) and zebrafish (Danio rerio) were presented with pairs of identical geometrical figures, one static and one physically rotating, and were trained to select the moving target in order to get a food reward. In the test phase, fish underwent non-rewarded probe trials in which they were presented with new pairs of stimuli, a RSI stimulus (Figure 1a) and a control stimulus that was identical to the illusory figure except that the luminance relationship was flipped between adjacent local texture elements (Figure 1b) so that the pattern does not induce illusory motion in humans ${ }^{16}$. In this situation, an animal perceiving a global rotation in the RSI stimulus is expected to select it rather than the counterpart that does not induce the motion illusion.

\section{Results}

Zebrafish. In the training phase, zebrafish showed a significant preference for the moving stimuli (one sample $\mathrm{t}$-test, $\mathrm{t}_{(11)}=7.27, p$ $<0.001)$. In the test phase, during which they were subjected to a series of non-rewarded probe trials (extinction trials) with the RSI and the control stimulus, zebrafish showed a clear choice of RSI compared with the control figure (one sample t-test, $\mathrm{t}_{(11)}=8.31, p$ $<0.001$ ) (Figure 3). For 10 out of 12 fish (83\%), the performance did not differ between illusory and real motion. For the remaining two fish, real motion was significantly more effective as illusory motion. Accuracy did not differ with respect to the two variants of RSI: four circle RSI (mean \pm SD: $0.61 \pm 0.06)$, nine circle RSI (0.61 \pm 0.07$)$; paired t-test, $\mathrm{t}_{(11)}=0.18, \mathrm{p}=0.853$.

Guppy. Similar results were observed in guppies. In the training phase, guppies showed a significant preference for the moving stimuli (one sample t-test, $\mathrm{t}_{(11)}=7.90, \mathrm{p}<0.001$ ). In the test phase, they showed a significant choice of RSI compared with the control figure (one sample t-test, $\mathrm{t}(11)=2.24, \mathrm{p}=0.046$ ) (Figure 3 ). For 8 out of 12 fish (67\%), illusory motion was equally effective as real motion. Real motion was significantly more effective for the four remaining fish.

Fish performance did not differ with respect to the two variants of RSI: four-circle RSI (mean \pm SD: $0.54 \pm 0.29)$, nine-circle RSI (0.62 $\pm 0.15)$; paired t-test, $\mathrm{t}_{(11)}=0.719, \mathrm{p}=0.487$.

No difference in the choice of RSI stimulus was found between the two species (independent t-test, $\mathrm{t}_{(22)}=0.30, \mathrm{p}=0.767$ ). See Table 1 to see the individual performance of each subject.

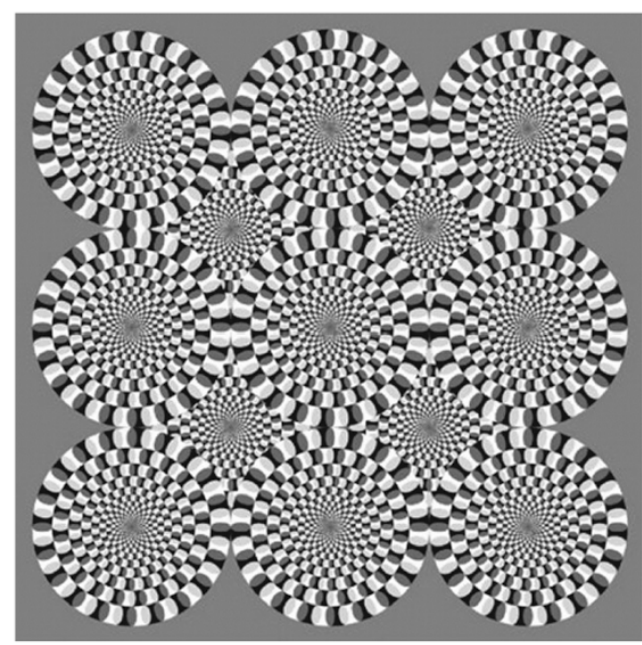

b

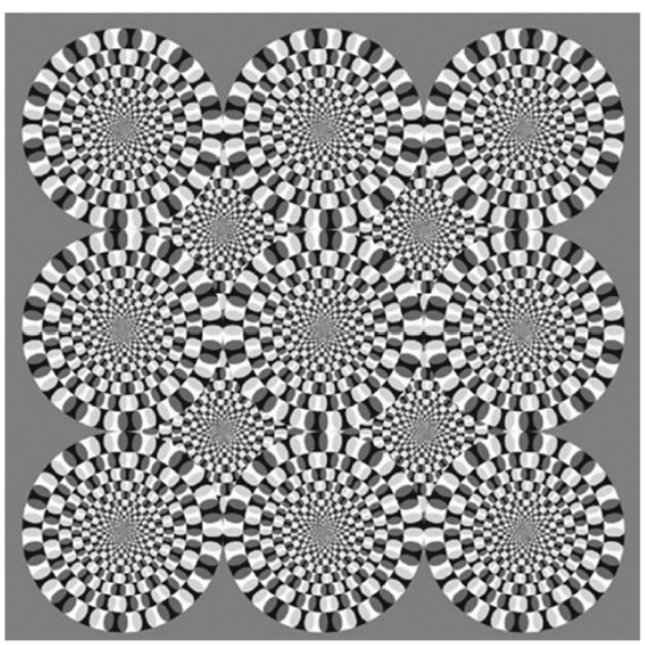

Figure $1 \mid$ The nine-circle version of the Rotating Snakes Illusion (RSI) (a) used in the test phase and its static control stimulus (b). Those stimuli were created by the authors of this article based on the original pattern by Kitaoka and Ashida ${ }^{13}$. 
Table 1 | Individual performance in the time spent near the moving target (training) and the Rotating Snake lllusion (test). The performance of 10 out of 12 zebrafish (83\%) and that of 8 out of 12 guppies $(67 \%)$ did not significantly differ between training and test phase

\begin{tabular}{|c|c|c|c|}
\hline SUBJECTS & TRAINING Mean \pm Std. dev. & TEST Mean \pm Std. dev. & $t$-test and $p$ value \\
\hline \multicolumn{4}{|l|}{ Zebrafish } \\
\hline 1 & $0.755 \pm 0.045$ & $0.626 \pm 0.111$ & $t(14)=2.20, p=0.045$ \\
\hline 2 & $0.765 \pm 0.031$ & $0.646 \pm 0.137$ & $\mathrm{t}(14)=1.67, p=0.117$ \\
\hline 4 & $0.723 \pm 0.091$ & $0.524 \pm 0.092$ & $t(14)=3.69, p=0.002$ \\
\hline 5 & $0.740 \pm 0.065$ & $0.586 \pm 0.175$ & $\mathrm{t}(14)=1.60, p=0.131$ \\
\hline 6 & $0.675 \pm 0.062$ & $0.600 \pm 0.144$ & $t(14)=0.95, p=0.359$ \\
\hline 9 & $0.630 \pm 0.042$ & $0.540 \pm 0.188$ & $t(14)=0.93, p=0.367$ \\
\hline 10 & $0.445 \pm 0.225$ & $0.602 \pm 0.096$ & $t(14)=2.10, p=0.054$ \\
\hline 11 & $0.656 \pm 0.228$ & $0.655 \pm 0.223$ & $t(14)=0.27, p=0.791$ \\
\hline 12 & $0.755 \pm 0.045$ & $0.626 \pm 0.111$ & $t(14)=0.61, p=0.552$ \\
\hline \multicolumn{4}{|l|}{ Guppy } \\
\hline 1 & $0.569 \pm 0.383$ & $0.657 \pm 0.235$ & $t(14)=0.99, p=0.342$ \\
\hline 2 & $0.812 \pm 0.112$ & $0.687 \pm 0.307$ & $\mathrm{t}(14)=0.57, \mathrm{p}=0.575$ \\
\hline 7 & $0.895 \pm 0.174$ & $0.629 \pm 0.296$ & $t(14)=1.81, p=0.091$ \\
\hline 8 & $0.487 \pm 0.563$ & $0.577 \pm 0.198$ & $\mathrm{t}(14)=1.01, p=0.330$ \\
\hline 9 & $0.945 \pm 0.037$ & $0.569 \pm 0.204$ & $t(14)=2.89, p=0.012$ \\
\hline 10 & $0.742 \pm 0.412$ & $0.426 \pm 0.262$ & $\mathrm{t}(14)=1.95, \mathrm{p}=0.071$ \\
\hline 11 & $0.543 \pm 0.531$ & $0.458 \pm 0.334$ & $t(14)=0.36, p=0.722$ \\
\hline 12 & $0.807 \pm 0.212$ & $0.446 \pm 0.256$ & $t(14)=2.29, p=0.038$ \\
\hline
\end{tabular}

\section{Discussion}

Our results demonstrated, for the first time, that a non-human species does perceive global rotary illusory motion that is elicited by a static pattern. Although fish diverged from land vertebrates approximately 450 million years ago and their visual system evolved in a very different ecological context, they are fooled by the RSI in a qualitative fashion that appears to be consistent with humans' perception of the same static figure.

In humans, two main physiological mechanisms seem to underlie the perception of motion observed in the RSI. Some studies reported convincing results supporting the role of fixational eye movements in triggering motion illusion ${ }^{16-18}$, while others stressed that the illusory effect originated in the visual cortex. Conway et al. ${ }^{20}$ reported that the differences in response latency to different contrast elements are responsible for the illusion and provided the first evidence that pairs of stimuli with the luminance sequence that characterizes the RSI can generate motion signals in the primary visual cortex (V1) and MT

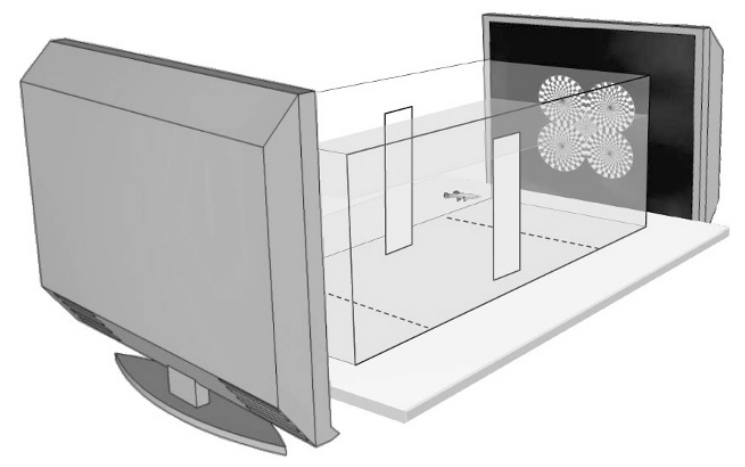

Figure $2 \mid$ Experimental apparatus. Fish were first trained with a food reward to discriminate between moving and non-moving targets displayed on two computer screens and hence tested in extinction with RSI stimulus and its static counterpart. direction-selective (DS) neurons. The two fMRI studies that directly investigated the $\mathrm{RSI}^{30,31}$ recorded an increase in neural activity in the motion-sensitive area of the human visual cortex during the observation of the RSI, suggesting that local motion signals in response to asymmetric spatial patterns at the level of V1 are integrated in the motion-sensitive cortical area (V5-MT complex) that creates the vivid rotatory motion perception. Global rotation is a peculiar type of motion that requires specifically V5-MT activity, no matter whether it is real ${ }^{32}$ or illusory ${ }^{21}$. The V5-MT complex possess neurons with large receptive fields that are able to integrate, into a global

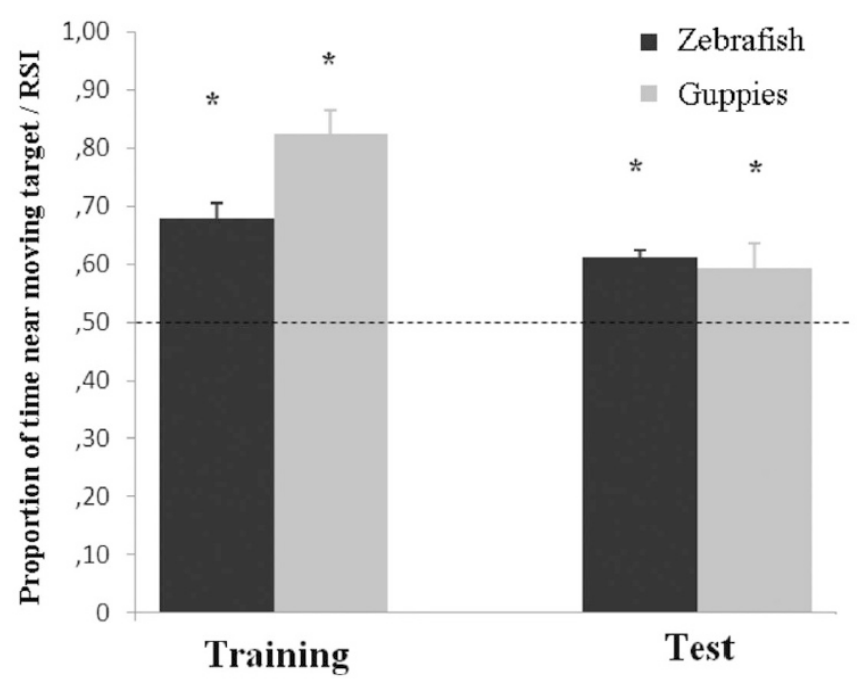

Figure $3 \mid$ Proportion of time spent by the fish ( $\mathrm{N}=12$ for each species) near the motion stimuli (training phase, 4 probe trials) and the RSI (test phase, 12 probe trials). ${ }^{*}=$ significant departure from chance level $(0.5)$, $\mathrm{p}<0.05$. 
motion, the local motion signals processed by the small receptive fields of the DS neurons populating V1.

In sum, both neurobiological and behavioral studies suggest a critical role of the visual cortex in the perception of motion in the RSI. Fish show eye movements that, together with head and body movements, probably produce motion signals on their retina that are comparable to those experienced in humans during eye movements ${ }^{33,34}$ but clearly do not have a cortex, and visual signals are processed by the optic tectum (OT). Therefore, different processes should be responsible for RSI in these organisms.

The motion percept is possibly elicited by stimulus processing in DS visual neurons in fish, too. These units respond optimally to stimulus motion in one (preferred) direction, but not in the opposite direction. DS cells were first described in the OT of the goldfish ${ }^{35}$ and also rabbits possess DS cells in their retina ${ }^{36}$. The DS ganglion cells (GC) in the retina are pure ON or OFF type and are characterized by small receptive fields. DS GC project to the OT. The DS neurons of the OT possess large receptive fields and collect signals from the retinal DS units of both ON and OFF subtypes with the same preferred direction ${ }^{37}$. One may argue that the fundamental mechanism that governs motion processing may have appeared early in vertebrate evolution and may still characterize today's fish. With appearance of the neocortex in the mammalian lineage, these mechanisms might have been co-opted by the visual cortical areas. A recent study, however, suggests that these old systems may still be in place, showing that these alternative pathways become apparent when the visual cortex is significantly damaged ${ }^{38}$. Blindsight patients, which are characterized by damage of the primary visual areas, were found to be able to perceive apparent motion by seemingly relying on a retinocollicular pathway, which bypasses V1, and directly reaches the MT/ V5 areas $^{38}$. Studying animals that have no cortex but exhibit perceptual behavior similar to human observers could be very useful to investigate mechanisms that were conserved in humans but are normally masked by the main modality of visual motion processing through the retino-geniculate-striate pathway.

In humans, across all ages, only $84 \%$ of the observers reported perceiving the RSI and 75\% reported perceived motion in the previous pattern devised by Fraser and Wilcox ${ }^{22,23}$. It is interesting to note that comparable percentages of subjects were found here to perceive illusory motion in both guppies and zebrafish. In humans, this trait is highly heritable ${ }^{22,24}$; it will be interesting to see if this polymorphism has a genetic basis in fish as well. A genetic contribution to individual differences in post-retinal neural processing has been shown for several other aspects of vision, including binocular rivalry ${ }^{39}$ and flicker fusion thresholds ${ }^{40}$, and contrast sensitivity ${ }^{41}$ variations in visual processing (perception and attention) have been associated with developmental disorders such as dyslexia and autism spectrum disorders ${ }^{42-48}$. Both guppies and zebrafish have been widely used for behavioral genetic studies. The zebrafish genome was one of the first to be sequenced; resources are available for the identification of gene function ${ }^{49}$, and the genome of the guppy is currently being sequenced $^{50}$. Numerous tools are available, especially for the former species, including several mutations that involve defects in the visual system $^{51}$. The discovery that fish respond to motion illusions adds to other evidence that fish and humans share many features of the visual system and makes these organisms an ideal model to study the neurobiology of motion perception.

From a phylogenetic point of view, guppies are distantly related to zebrafish. According to recent estimates ${ }^{52}$, zebrafish and guppies belong to clades (Ostariophysi and Acanthopterygii, respectively), which diverged more than 250 million years ago. This suggests that the RSI perception might be a general feature of a teleost visual system. Comparative data from other vertebrate and invertebrate classes are now needed in order to determine whether this is a convergent trait that evolved independently in fish and primate lineages or whether all vertebrates, and even some invertebrates, share the same basic processes of motion perception.

In conclusion, our evidence demonstrates that the visual system of fish is clearly equipped with neuronal mechanisms capable of mediating RSI and challenges the idea that higher-level, cortical mechanisms are strictly necessary to elicit illusory motion. It seems plausible that the large receptive fields of the ON/OFF DS visual neurons, populating the OT, could serve as the neural substrate to process global illusory motion in the absence of a cortex. However, our results do not necessarily contradict the literature showing that humans need a cortex to perceive global illusory motion. Rather, we suggest that our findings and those from blindsight patients ${ }^{38}$ agree in indicating that, as shown for numerous other visual functions, the general principles that underlie motion perception appeared early in vertebrate evolution and have been conserved in the mammalian lineage even after many visual functions became localized in the neocortex. If confirmed, this would open up the possibility of developing useful animal models to study the genetic and neural bases of motion perception.

\section{Methods}

Subjects. Two species of teleosts were used in this study: zebrafish (Danio rerio), a leading model organism for studies in neurobiology of vision and

neurodevelopmental genetics ${ }^{51}$, and guppy (Poecilia reticulata), a leading model for behavioral studies in fish ${ }^{53}$. Twelve adult female zebrafish and twelve adult female guppies were tested in our experiment. The two species were maintained separately in 150 one-stock aquaria (approximately 15 individuals per aquarium). Aquaria were provided with natural gravel, an air filter, and live plants. Both stock aquaria and experimental tanks were maintained at a constant temperature of $25 \pm 1{ }^{\circ} \mathrm{C}$ and a $14: 10 \mathrm{~h}$ light: dark (L:D) photoperiod with an 18-W fluorescent light. Before the experiment, fish were fed twice daily to satiation with commercial food flakes and live brine shrimp (Artemia salina).

Apparatus and Stimuli. We use a modification of the procedures that have been traditionally adopted for investigating visual discrimination in fishes ${ }^{54}$, a twoalternative spatial forced-choice paradigm that was recently developed by Agrillo et al. ${ }^{55,56}$ to study numerical competence in fish.

The experimental apparatus was composed by a $50 \times 19 \times 32 \mathrm{~cm}$ tank. It was filled with gravel and $24 \mathrm{~cm}$ of water. The long walls were covered with green plastic material, and the short walls were covered with white plastic material. To reduce the potential effects of social isolation ${ }^{57}$, two mirrors $(29 \times 5 \mathrm{~cm})$ were placed in the middle of the tank, $3 \mathrm{~cm}$ away from the long walls. At the two short ends of the tank, two identical monitors (19 inches) were used to present the stimuli.

The stimuli employed in the training phase were pairs of identical geometrical figures $(12 \times 12 \mathrm{~cm})$ presented on a computer screen. Each array contained circles, stars, triangles and rectangles presented in two different conditions: in the former ('moving target'), the items rotated in a clockwise or counterclockwise direction at three different speeds $(5 / 15 / 21 \mathrm{rpm})$; in the latter condition, the same figures were presented without any movement (non-moving target). To avoid any issue related to potential difference in color vision of the two species, all stimuli were converted to grayscale (as the RSI and the control stimulus presented in the test phase).

A total of 16 different couples of stimuli were employed and alternated in a pseudorandom order. In particular in the initial training, arrays containing a single figure (either static or dynamic) was presented. Subsequently, in order to familiarize fish with the complex pattern of the RSI, multiple figures were presented within each array (see Supplemental Material for examples). Moving and non-moving targets were created and presented through Adobe Flash CS4 ${ }^{\circledR}$.

In test trials, we presented the Rotating Snakes Illusion ${ }^{13}$ (Figure 1a). A grey-scale version of the $\mathrm{RSI}^{13}$ and its control (both encompassed in a $12 \times 12 \mathrm{~cm}$ square) were presented in the test phase. The latter stimulus was identical to the illusory figure, except that the luminance relationship was flipped between adjacent local texture elements (Figure 1a and b). This pattern does not induce illusory motion in humans ${ }^{16}$. Two different versions were created for both stimuli: 4 or 9 circles.

Six identical experimental tanks were employed. They were placed close to each other on the same table and lit by two fluorescent lamps (36 W). A video camera was suspended about 1 meter above the experimental tanks and used to record the position of the subjects during the tests.

Procedure. The experiment was divided into two different phases: training and test.

Training. To familiarize the fish with the novel environment, in the nine days preceding the training, six subjects were simultaneously inserted in a tank similar to that used in the experiment but enlarged in size $(60 \times 40 \times 35 \mathrm{~cm})$. During the first day of this pre-training phase, subjects were exposed together to a continuous change of the monitors' background color (from black to white alternatively). This lasted for 6 hours, during which the background color changed every hour. The second day, fish were moved in couples into the experimental tanks and underwent the same 
treatment. After this phase, the subjects returned to their home tank for 5 days; then the same pre-training steps were repeated, one day in the enlarged tank and another day in the experimental tanks.

After this period, the fish started the training phase. During this phase, they received four trials per day (three consecutive days, for a total of 12 trials). Soon after the stimuli appeared on the screens, the experimenter used a Pasteur pipette to release the food reward (brine shrimps) in correspondence with the moving stimulus; an identical syringe was used to simultaneously insert pure water close to the still stimulus. Subjects were left free to feed for seven minutes. After this time, the stimuli disappeared from the screen. The inter-trial interval lasted three hours. Stimuli were presented in a pseudo-random sequence and the left-right positions of the stimuli were counterbalanced over trials.

In order to assess task learning, on days 4 and 5, two probe trials were alternated each day with two reinforced trials (four probe trials in total). In the probe trials, stimuli appeared on the screen for four minutes; no reinforcement was provided (extinction procedure) and the time spent by fish within $12 \mathrm{~cm}$ ('choice area', depicted as a dotted line in Figure 2) in correspondence to the stimuli was recorded as a measure of their capacity to discriminate between them. Reinforced trials were identical to those described for days 1 to 3 . To avoid the possibility of fish using the local/spatial cues of their tank, each subject was moved from one tank to another at the end of each day.

Test. After a two-day interval, three probe trials were presented each day for four consecutive days (days 8-11). The RSI was contrasted with its control stimulus. The inter-trial interval lasted three hours. The three probe trials were alternated with two reinforced trials in which we presented the same stimuli of the training phase (true moving vs. static targets), according to the following sequence: probe trial/reinforced trial/probe trial/reinforced trial/probe trial. The proportion of time spent near the RSI during probe trials was recorded as the dependent variable. Frequencies were arcsine (square root)-transformed ${ }^{58}$.

In order to test if there could be other cues in the RSI stimulus, not related to motion, that might attract the subjects to this stimulus; or, vice versa, any other cue in the static control stimulus that might repel fish, we also ran a control experiment. Twelve naïve fish, six guppies and six zebrafish, were singly housed in tanks similar to those used in the main experiment and acclimated for $24 \mathrm{~h}$. Fish were then simultaneously exposed to the RSI and the control stimuli and we recorded the position for 20 minutes. After a 2-hour interval, we repeated the test, switching the position of the stimuli. We computed the time spent within $20 \mathrm{~cm}$ from the stimuli. Zebrafish spent $51.9 \pm 4.9 \%$ and guppies $51.8 \pm 8.5 \%$ near the RSI stimulus. There was no significant preference/repulsion for any of the stimuli (Repeated Measure ANOVA, F(1,10) = $0.411, \mathrm{P}=0.536)$, no species difference: $(\mathrm{F}(1,10)=3.224, \mathrm{P}=0.103)$ or interaction $(\mathrm{F}(1,10)=0.053, \mathrm{P}=0.823)$

The experiments comply with all laws of the country (Italy) in which they were performed (D.M. 116192) and the study was approved by the 'Ministero della Salute' (permit number: 6726-2011). The methods were carried out in accordance with the approved guidelines.

1. Shichida, Y. \& Matsuyama, T. Evolution of opsins and phototransduction. Proceedings of the Royal Society of London. Series B. Biological Sciences 364, 2881-2895. (2009)

2. Hubel, D. H. \& Wiesel, T. N. Receptive fields of single neurones in the cat's striate cortex. The Journal of physiology 148, 574-591 (1959).

3. Hubel, D. H. \& Wiesel, T. N. Ferrier Lecture: Functional Architecture of Macaque Monkey Visual Cortex. Proceedings of the Royal Society of London. Series B. Biological Sciences 198, 1-59, doi:10.1098/rspb.1977.0085 (1977).

4. Eagleman, D. M. Visual illusions and neurobiology. Nature Reviews Neuroscience 2, 920-926 (2001)

5. Nieder, A. Seeing more than meets the eye: processing of illusory contours in animals. Journal of Comparative Physiology A 188, 249-260 (2002).

6. Kelley, L. A. \& Kelley, J. L. Animal visual illusion and confusion: the importance of a perceptual perspective. Behavioral Ecology, art118 (2013).

7. Pinna, B. \& Brelstaff, G. J. A new visual illusion of relative motion. Vision Research 40, 2091-2096 (2000).

8. Gori, S. \& Hamburger, K. A new motion illusion: the Rotating-Tilted-Lines illusion. Perception 35, 853-857 (2006)

9. Gori, S. \& Yazdanbakhsh, A. The riddle of the Rotating-Tilted-Lines illusion. Perception 37, 631-635 (2008).

10. Gori, S., Giora, E. \& Stubbs, D. A. Perceptual compromise between apparent and veridical motion indices: The Unchained-Dots illusion. Perception 39, 863 (2010).

11. Gori, S., Giora, E., Yazdanbakhsh, A. \& Mingolla, E. A new motion illusion based on competition between two kinds of motion processing units: The Accordion Grating. Neural networks 24, 1082-1092 (2011)

12. Yazdanbakhsh, A. \& Gori, S. Mathematical analysis of the Accordion Grating illusion: a differential geometry approach to introduce $3 \mathrm{D}$ aperture problem. Neural networks 24, 1093-1101 (2011).

13. Kitaoka, A. \& Ashida, H. Phenomenal characteristics of the peripheral drift illusion. Vision 15, 261-262 (2003).

14. Gori, S., Hamburger, K. \& Spillmann, L. Reversal of apparent rotation in the Enigma-figure with and without motion adaptation and the effect of T-junctions. Vision research 46, 3267-3273 (2006).
15. Spillmann, L. The Ōuchi-Spillmann illusion revisited. Perception 42, 413-429 (2013).

16. Murakami, I., Kitaoka, A. \& Ashida, H. A positive correlation between fixation instability and the strength of illusory motion in a static display. Vision research 46, 2421-2431 (2006)

17. Troncoso, X. G., Macknik, S. L., Otero-Millan, J. \& Martinez-Conde, S. Microsaccades drive illusory motion in the Enigma illusion. Proceedings of the National Academy of Sciences 105, 16033-16038 (2008).

18. Otero-Millan, J., Macknik, S. L. \& Martinez-Conde, S. Microsaccades and blinks trigger illusory rotation in the "Rotating Snakes" illusion. The Journal of Neuroscience 32, 6043-6051 (2012).

19. Zeki, S., Watson, J. \& Frackowiak, R. S. Going beyond the information given: the relation of illusory visual motion to brain activity. Proceedings of the Royal Society of London. Series B: Biological Sciences 252, 215-222 (1993).

20. Conway, B. R., Kitaoka, A., Yazdanbakhsh, A., Pack, C. C. \& Livingstone, M. S. Neural basis for a powerful static motion illusion. The Journal of neuroscience $\mathbf{2 5}$ 5651-5656 (2005).

21. Ruzzoli, M. et al. The neural basis of the Enigma illusion: A transcranial magnetic stimulation study. Neuropsychologia 49, 3648-3655 (2011).

22. Fraser, A. \& Wilcox, K. J. Perception of illusory movement. Nature 281, 565-566 (1979).

23. Billino, J., Hamburger, K. \& Gegenfurtner, K. R. Age effects on the perception of motion illusions. Perception 38, 508 (2009).

24. Gori, S. et al. The DCDC2 intron 2 deletion impairs illusory motion perception unveiling the selective role of magnocellular-dorsal stream in reading (dis)ability. Cerebral Cortex (in press).

25. Scott, T. R. \& Powell, D. A. Measurement of a visual motion aftereffect in the rhesus monkey. Science 140, 57-59 (1963).

26. Srinivasan, M. V. \& Dvorak, D. The waterfall illusion in an insect visual system. Vision research 19, 1435-1437 (1979).

27. Xiao, Q. \& Güntürkün, O. Do pigeons perceive the motion aftereffect? A behavioral study. Behavioural brain research 187, 327-333 (2008).

28. Tuthill, J. C., Chiappe, M. E. \& Reiser, M. B. Neural correlates of illusory motion perception in Drosophila. Proceedings of the National Academy of Science USA. 108, 9685-9690 (2011).

29. Orger, M. B., Smear, M. C., Anstis, S. M. \& Baier, H. Perception of Fourier and non-Fourier motion by larval zebrafish. Nature neuroscience 3, 1128-1133 (2000).

30. Kuriki, I., Ashida, H., Murakami, I. \& Kitaoka, A. Functional brain imaging of the Rotating Snakes illusion by fMRI. Journal of Vision 8, 16 (2008).

31. Ashida, H., Kuriki, I., Murakami, I., Hisakata, R. \& Kitaoka, A. Direction-specific fMRI adaptation reveals the visual cortical network underlying the "Rotating Snake" illusion. Neuroimage 61, 1143-1152 (2012).

32. Morrone, M. et al. A cortical area that responds specifically to optic flow, revealed by fMRI. Nature neuroscience 3, 1322-1328 (2000).

33. Portugues, R. \& Engert, F. The neural basis of visual behaviors in the larval zebrafish. Current opinion in neurobiology 19, 644-647 (2009).

34. Maurer, C. M., Huang, Y.-Y. \& Neuhauss, S. C. Application of zebrafish oculomotor behavior to model human disorders. Reviews in the Neurosciences 22 , 5-16 (2011).

35. Cronly-Dillon, J. Units sensitive to direction of movement in goldfish optic tectum. (1964) Nature. 203, 214-215 (1964)

36. Barlow, H. B. \& Hill, R. M. Selective sensitivity to direction of movement in ganglion cells of the rabbit retina. Science. 139, 412-414 (1963).

37. Maximov, V., Maximova, E. \& Maximov, P. Direction selectivity in the goldfish tectum revisited. Annals of the New York Academy of Sciences 1048, 198-205 (2005).

38. Azzopardi, P. \& Hock, H. S. Illusory motion perception in blindsight. Proceedings of the National Academy of Sciences 108, 876-881 (2011).

39. Miller, S. M. et al. Genetic contribution to individual variation in binocular rivalry rate. Proceedings of the National Academy of Sciences 107, 2664-2668 (2010).

40. Vogel, F., Schalt, E., Krüger, J., Propping, P. \& Lehnert, K. F. The electroencephalogram (EEG) as a research tool in human behavior genetics: Psychological examinations in healthy males with various inherited EEG variants. Human Genetics 47, 1-45, doi:10.1007/BF00295569 (1979).

41. Cronin-Golomb, A. et al. Genetic influence on contrast sensitivity in middle-aged male twins. Vision research 47, 2179-2186 (2007).

42. Stein, J. \& Walsh, V. To see but not to read; the magnocellular theory of dyslexia. Trends in neurosciences 20, 147-152 (1997).

43. Franceschini, S., Gori, S., Ruffino, M., Pedrolli, K. \& Facoetti, A. A causal link between visual spatial attention and reading acquisition. Current Biology 22, 814-819 (2012)

44. Franceschini, S. et al. Action video games make dyslexic children read better. Current Biology 23, 462-466 (2013).

45. Ronconi, L., et al. Decreased Coherent Motion Discrimination in Autism Spectrum Disorder: The Role of Attentional Zoom-out Deficit. Plos One 7, e49019 (2012).

46. Ronconi, L., Gori, S., Ruffino, M., Molteni, M. \& Facoetti, A. Zoom-out attentional impairment in children with autism spectrum disorder. cortex 49, 1025-1033 (2013).

47. Gori, S. \& Facoetti, A. Perceptual learning as a possible new approach for remediation and prevention of developmental dyslexia. Vision research $99,78-87$ (2014). 
48. Gori, S., Cecchini, P., Bigoni, A., Molteni, M. \& Facoetti, A. Magnocellular-dorsal pathway and sub-lexical route in developmental dyslexia. Frontiers in human neuroscience 8, 460 (2014)

49. Bradford, Y. et al. ZFIN: enhancements and updates to the Zebrafish Model Organism Database. Nucleic acids research 39, D822-D829 (2011)

50. Fraser, B. A., Weadick, C. J., Janowitz, I., Rodd, F. H. \& Hughes, K. A. Sequencing and characterization of the guppy (Poecilia reticulata) transcriptome. BMC genomics 12, 202 (2011).

51. Neuhauss, S. C. et al. Genetic disorders of vision revealed by a behavioral screen of 400 essential loci in zebrafish. The Journal of neuroscience 19, 8603-8615 (1999).

52. Steinke, D., Salzburger, W. \& Meyer, A. Novel relationships among ten fish model species revealed based on a phylogenomic analysis using ESTs. Journal of molecular evolution 62, 772-784 (2006).

53. Magurran, A. E. Evolutionary ecology: the Trinidadian guppy. (Oxford University Press, 2005).

54. Sovrano, V. A. \& Bisazza, A. Recognition of partly occluded objects by fish. Animal cognition 11, 161-166 (2008).

55. Agrillo, C., Miletto Petrazzini, M. E., Piffer, L., Dadda, M. \& Bisazza, A. A new training procedure for studying discrimination learning in fish. Behavioural brain research 230, 343-348 (2012).

56. Agrillo, C., Miletto Petrazzini, M. E., Tagliapietra, C. \& Bisazza, A. Inter-specific differences in numerical abilities among teleost fish. Frontiers in psychology 3 (2012).

57. Miletto Petrazzini, M. E., Agrillo, C., Piffer, L., Dadda, M. \& Bisazza, A. Development and application of a new method to investigate cognition in newborn guppies. Behavioural brain research 233, 443-449 (2012).

58. Sokal, R. R. \& Rohlf, F. J. Biometry: The principles and practice of statistics in biological research. (Freeman and Company, 1995).

\section{Acknowledgments}

The authors would like to thank Michael J. Beran and Lothar Spillmann for valuable comments and suggestions, and Luca Leoni for his help conducting the experiment. This study was supported by research grants from the University of Padua to Angelo Bisazza and by FIRB grant (RBFR13KHFS) from the 'Ministero dell'Istruzione, Università e Ricerca' (MIUR) to Christian Agrillo.

\section{Author contributions}

All authors (S.G., C.A., M.D., A.B.) designed the study, set up the procedure, analyzed the data, and wrote the paper.

\section{Additional information}

Supplementary information accompanies this paper at http://www.nature.com/ scientificreports

Competing financial interests: The authors declare no competing financial interests.

How to cite this article: Gori, S., Agrillo, C., Dadda, M. \& Bisazza, A. Do Fish Perceive Illusory Motion?. Sci. Rep. 4, 6443; DOI:10.1038/srep06443 (2014).

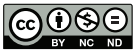

This work is licensed under a Creative Commons Attribution-NonCommercialNoDerivs 4.0 International License. The images or other third party material in this article are included in the article's Creative Commons license, unless indicated otherwise in the credit line; if the material is not included under the Creative Commons license, users will need to obtain permission from the license holder in order to reproduce the material. To view a copy of this license, visit http:// creativecommons.org/licenses/by-nc-nd/4.0/ 